\title{
BILATERAL OPEN TYPE IIIB INJURIES- "FLOATING FOREARM" WITH CONTRALATERAL DISTAL RADIUS PHYSEAL SEPARATION- A RARE CASE REPORT
}

\author{
Naba Pallab Chetia1, Manasjyoti Das ${ }^{2}$
}

${ }^{1}$ Assistant Professor, Department of Orthopaedics, Assam Medical College and Hospital, Dibrugarh, Assam.

2 Postgraduate Trainee, Department of Orthopaedics, Assam Medical College and Hospital, Dibrugarh, Assam.

HOW TO CITE THIS ARTICLE: Chetia NP, Das M. Bilateral open type IIIB injuries- "Floating Forearm" with contralateral distal radius physeal separation- a rare case report. J. Evolution Med. Dent. Sci. 2018;7(01):125-128, DOI: 10.14260/jemds/2018/27

\section{PRESENTATION OF CASE}

A ten-year-old boy presented to our emergency department with multiple injuries to both upper limbs following fall to the ground on outstretched hands from a mango tree of around 20 feet high.

He had lacerated wounds on the anterior aspect of both the wrists, each measuring around $4 \mathrm{~cm} \times 4 \mathrm{~cm}$ size. The distal radius metaphyseal end of the proximal fragment was seen protruding out through the wound and were devoid of any soft tissue or periosteum attachment. The exposed bone ends were contaminated with mud. Also, there was grossly swollen and deformed looking right elbow with restricted active and passive movements. There was no neurovascular deficit. Other skeletal, head, chest and abdominal injuries were ruled out.

The patient was administered IV fluids, tetanus toxoid, anti-tetanus immunoglobulins and analgesics. Swabs from both the wounds were collected for culture and sensitivity of potentially contaminating micro-organisms and then a dose of broad-spectrum IV antibiotic was administered. Thorough cleaning of the wound and exposed bone ends was performed by using copious amount of normal saline and both the limbs were splinted. Once the patient was haemodynamically stabilised, anteroposterior and lateral x-rays of both the forearms with wrist and elbow joints and also one exclusively dedicated for the right elbow were obtained. Clinicoradiologically, diagnosis of Gustilo-Anderson Type IIIB Open Bilateral Distal Radius Physeal Separation (SalterHarris type I) with greenstick fracture of right distal ulna was established. Due to pain and non-compliance in positioning of the injured limb on $\mathrm{x}$-ray table, a true AP and lateral views of the right elbow could not be obtained and therefore exact radiological diagnosis of the right elbow injury could not be ascertained at emergency department.

The patient was taken to emergency surgical intervention. As our customary practice, we imaged the injured limbs of the anaesthetised patient under C-arm before proceeding for surgery. The right elbow injury could then only be diagnosed as anterior elbow dislocation, which we could not ascertain earlier in preoperative x-rays. The open wounds were surgically extended for the ease of thorough inspection and debridement.

'Financial or Other Competing Interest': None.

Submission 26-10-2017, Peer Review 12-12-2017,

Acceptance 18-12-2017, Published 01-01-2018.

Corresponding Author:

Dr. Manasjyoti Das,

Department of Orthopaedics,

Assam Medical College and Hospital,

Dibrugarh- 786002, Assam.

E-mail: manasjyotidr@gmail.com

DOI: $10.14260 /$ jemds $/ 2018 / 27$

\section{(c) (1) (3) $\Theta$}

Both the distal radius were anatomically reduced through the open wounds. Under C-arm fluoroscopy guidance, reduction was confirmed and fixed with two smooth thin sharp K-wires placed parallel to each other. Pins were placed from the distal epiphysis of the radial styloid obliquely across the physis into the more proximal ulnar aspect of the radial metaphysis. Closed reduction of the right elbow anterior dislocation was unsuccessful despite several gentle attempts and therefore open reduction was done and fixed with a transolecranon K-wire to the distal humerus to hold the reduction. A below elbow $\mathrm{POP}$ splint on the left and an above elbow POP splint on the right were applied.

Postoperatively, neurovascular status of both upper limbs was normal. IV antibiotics, anti-inflammatory drugs and analgesics were continued. Both the upper limbs were elevated and active as well as assisted exercises for finger of both sides and left elbow was encouraged. The open wounds healed by secondary intention on regular antiseptic and aseptic dressing. All the K-wires were removed at 4 weeks postoperatively. The patient was rehabilitated to range of motion and strengthening exercise under physiotherapist.

\section{DIFFERENTIAL DIAGNOSIS}

The differential diagnosis of right elbow injury included fractures of the supracondylar area, medial condyle or lateral condyle of humerus, elbow dislocation, Monteggia or Monteggia variant fracture dislocation.

The differential diagnosis of the open injuries of bilateral distal forearm included physeal separation of distal radius as well as distal end diaphyseal fractures.

\section{CLINICAL DIAGNOSIS}

As the fractured bone ends of both distal forearm were visible and palpable through the open wounds, the clinical high index of suspicion was that of physeal injury of bilateral distal radius. As both the distal forearm bony injuries were associated with extensive soft tissue damage with presence of significant periosteal stripping, they were classified as Gustilo-Anderson type IIIB open fractures.

The severe pain deterred the child from cooperating with us for examination of his injured right elbow, especially active and passive movement of flexion and extension. As there was gross swelling of right elbow, the three bony points of lateral and medial epicondyle and tip of the olecranon could not be palpated even with great difficulty. Under these limiting circumstances, the very important clinical examination of relationship of "three bony points around the elbow" could not be ascertained. Therefore, the possibility of supracondylar fracture of humerus (which is the most common fracture around the elbow in children) and elbow dislocation were kept in mind. However, they could not be differentiated on clinical basis. 


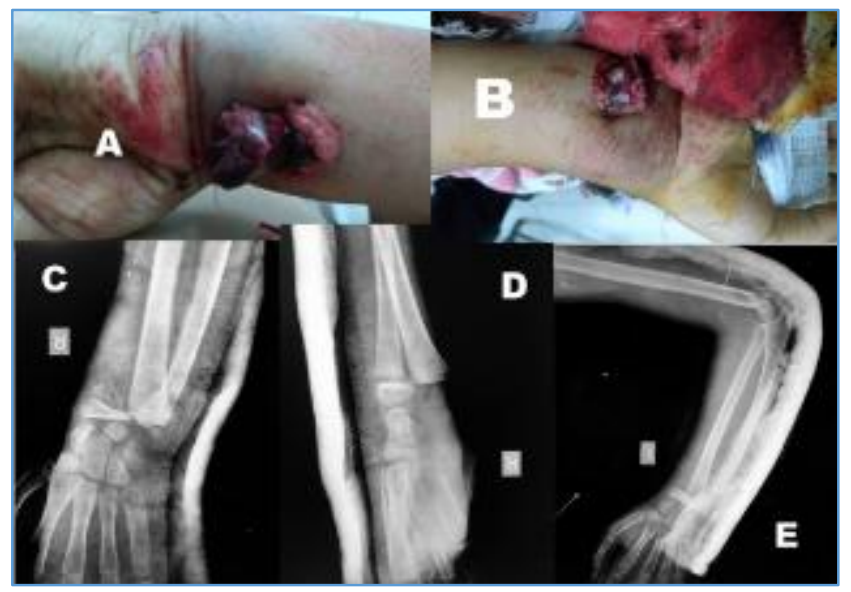

Figure 1A and B: Open Distal Radius Physeal Injury of Bilateral Wrists. C and D: AP and Lateral X-Ray Views Type I Salter-Harris Injury of Right Distal Radius. E: AP X-Ray View of Salter-Harris Type I Physeal Injury Left Distal Radius

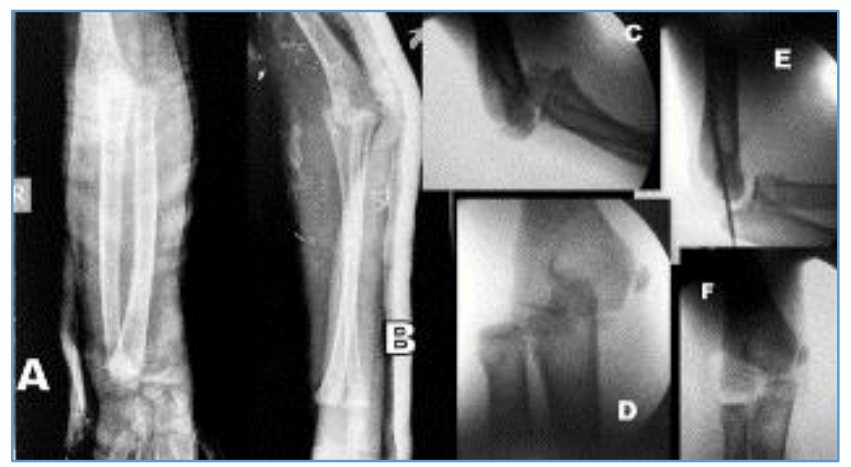

Figure 2A and B: Inconclusive X-Ray Views of Right Forearm for Diagnosis of Anterior Elbow Dislocation. $C$ and D: Diagnosis of Anterior Elbow Dislocation of Right Side established by C-Arm Views. E and F: Reduction of Right Anterior Elbow Dislocation in C-Arm

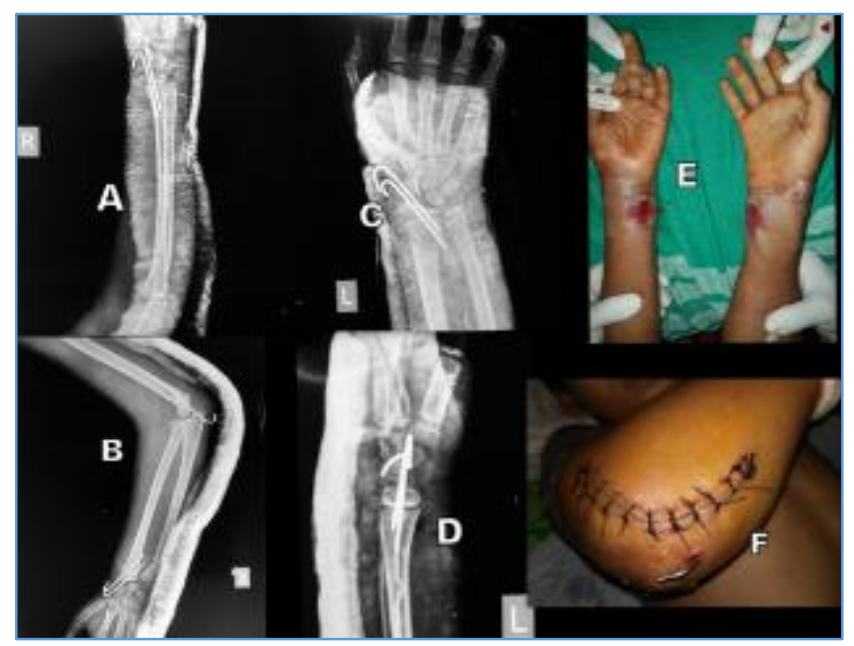

Figure 3: A and B: X-ray of the Right Forearm showing reduced Elbow Dislocation and reduced Distal Radius Physeal Injury with $K$-wires in situ. C and D: X-ray of the Left Wrist showing reduced Distal Radius Physeal Injury with $K$-wires in situ. E: Open Wounds on Bilateral Wrists in the Process of secondary Healing. F: Surgical Wound over the Right Elbow.

\section{DISCUSSION OF MANAGEMENT}

Simultaneous ipsilateral fracture dislocation of the elbow and fracture of the forearm termed as "floating forearm" is an extremely rare injury in children.[1] Distal radial physeal fractures are far more common than distal ulnar fractures. ${ }^{2]}$ Open physeal fractures are rare.[3] Open bilateral distal radial physeal fractures are not commonly documented in the literature. Anterior elbow dislocations are also rare in children. Of the 317 elbows in the combined series ${ }^{[4]}$ only 5 were anterior, for an incidence of slightly over $1 \%$. Our case assumes importance because of the combination of the rare injuries of open bilateral distal radial physeal fractures with anterior elbow dislocation on the right side constituting floating forearm injury, which are not commonly reported in the literature.

Distal radial physeal fractures most commonly affect nondominant arm in boys with peak incidence in preadolescent growth spurt. ${ }^{[2]}$ More than $50 \%$ of them have an associated ulnar fracture, which is usually an ulnar styloid fracture but can be a distal ulnar plastic deformation, greenstick or complete fracture.[5] In our case also, there was greenstick fracture of the distal end of ulna on right side.

Percutaneous pin fixation is one of the methods of treatment in a displaced radial physeal fracture with neurovascular compromise and significant volar soft tissue swelling[6] as well as in "floating elbow" injuries.[3] These patients are at risk for development of an acute carpal tunnel syndrome or forearm compartment syndrome with closed reduction and well-moulded cast.[6,7] The torn periosteum volarly allows the fracture bleeding to dissect into the volar forearm compartments and carpal tunnel. If a tight cast is applied with a volar mould over that area, compartment pressures can increase dangerously. Percutaneous pin fixation allows the application of a loose splint or cast without the risk of loss of fracture reduction. In addition, acute percutaneous pinning of the fracture prevents increased swelling, cast splitting and concerns about malunion or growth arrest with repeat reduction.

Pin fixation can be either single or double. Pin is placed from the distal epiphysis of the radial styloid obliquely across the physis into the more proximal ulnar aspect of the radial metaphysis. Often in children and adolescents, a single pin and the reduced periosteum provide sufficient stability to prevent re-displacement of the fracture. If fracture stability is questionable with a single pin, a second pin should be placed which can either parallel the first pin as we performed in our case or to create cross-pin stability, can be placed distally from the ulnar corner of the radial epiphysis between the fourth and fifth dorsal compartments and passed obliquely to the proximal radial portion of the metaphysis. Splint or cast immobilisation is used but does not need to be tight, because fracture stability is provided by the pins. The pins are left in until there is adequate fracture healing (usually 4 weeks).[8]

Cast immobilisation is discontinued when there is clinical and radiographic evidence of fracture healing, generally 4 to 6 weeks after fracture. As the risk of post-traumatic physeal disturbance is approximately $4 \%$ to $5 \%$, follow-up radiographs are obtained at 6 to 12 months after fracture to be certain there is no growth arrest.[9]

The anterior dislocation of elbow is rare as compared with posterior dislocation but are associated with increased 
incidence of complications, such as brachial artery rupture or thrombosis and associated fractures.[10,11,12] Although, anterior elbow dislocations usually are caused by a direct blow to the posterior aspect of the flexed elbow, ${ }^{[6]}$ yet hyperextension of the elbow also has been implicated.[12]

Closed reduction is usually accomplished by flexing the elbow and pushing the forearm proximally and downward at the same time.[13] To make reduction easier, the distal humerus can be forced in an anterior direction by pushing on the posterior aspect of the distal arm. Surgery usually is not required unless the dislocation is open, associated brachial artery injury or associated fracture that does not realign satisfactorily after closed reduction. ${ }^{[12,14]}$ In our case as we failed to achieve closed reduction with multiple attempts of gentle manipulation, we needed to do ORIF. Because most anterior dislocations occur in flexion, the elbow should be immobilised in some extension for 1 to 3 weeks followed by protected active range of motion exercises.

Results of paediatric fracture dislocations of the elbow are generally perceived as reasonable with internal fixation of concomitant bony avulsions, but case reports of elbow stiffness after simple elbow trauma have drawn the focus on the difficulty to treat sequelae after paediatric elbow fracture dislocation.[15-20] Stability of the ulnohumeral joint after elbow trauma is dependent on both bony and soft tissue constraints.[21,22,23] In young children it is rare and associated with high-energy trauma.[24,15] Disruption of osseous and/or articular stabilisers dramatically increases the risk of redislocation and recurrent instability.[17,19,20] As even simple dislocation of the paediatric elbow or single-column injuries of either the lateral or medial epicondyle are reported to lead to both deformity and loss of function. $[15,16,18,25]$

Floating forearm is a rare surgical emergency. Early diagnosis with anatomic reduction provides satisfactory functional results. In addition to open surgical treatment in the emergency, post-operative rehabilitation is essential.[25]

\section{FINAL DIAGNOSIS}

Clinicoradiologically, a final diagnosis of Gustilo-Anderson Type IIIB Open Bilateral Distal Radius Physeal Separation (Salter-Harris type I) with green stick fracture of right distal ulna with anterior dislocation of right elbow, constituting right-sided "floating forearm injury" with intact distal neurovascular status following alleged fall from height in a ten-year-old boy was established.

\section{REFERENCES}

[1] Gausepohl T, Mader K, Kirchner S, et al. The "floating forearm" injury in a child: a case report. Strat Traum Limb Recon 2007;2(1):48-54.

[2] Landin LA. Fracture patterns in children. Analysis of 8,682 fractures with special reference to incidence, etiology and secular changes in a Swedish urban population 1950-1979. Acta Orthop Scand Suppl 1983;202:1-109.

[3] Schoenecker JG, Bae DS. Fractures of the distal radius and ulna. In: (Jack) Flynn JM, Skaggs DL, Waters PM. eds. Rockwood and Wilkin's Fractures in Children. $8^{\text {th }}$ edn. Philadelphia, PA: Wolters \& Kluwer Health 2015;349-441.
[4] Linscheid Rl, Wheeler DK. Elbow dislocations. JAMA 1965;194(11):1171-6.

[5] Lesko PD, Georgis T, Slabaugh P. Irreducible Salter Harris type II fracture of the distal radial epiphysis. J Pediatr Orthop 1987;7(6):719-21.

[6] Waters PM, Kolettis GJ, Schwend R. Acute median neuropathy following physeal fractures of the distal radius. J Pediatr Orthop 1994;14(2):173-7.

[7] Crawford AH. Pitfalls and complications of fractures of the distal radius and ulna in childhood. Hand Clin 1988;4(3):403-13.

[8] Prasad K, Dayanandam B, Gakhar H, et al. Concomitant elbow and perilunate dislocation: floating forearm. Internet J Orthop Surg 2008;8(1)1-4.

[9] Cannata G, De Maio F, Mancini F, et al. Physeal fractures of the distal radius and ulna: long-term prognosis. J Orthop Trauma 2003;17(3):172-9.

[10] Jackson JA. Simple anterior dislocation of the elbow joint with rupture of the brachial artery. Case report. Am J Surg 1940;47:479-86.

[11] Spear HC, Janes JM. Rupture of the brachial artery accompanying dislocation of the elbow or supracondylar fracture. J Bone Joint Surg Am 1951;33$A(4): 889-94$.

[12] Wilkerson RD. Anterior elbow dislocation associated with olecranon fractures. Review of the literature and case report. Iowa Onthop J 1993;13:223-5.

[13] Anthony A, Stans J, Lawrence TR. Dislocations of the elbows, medial epicondylar humerus. In: (Jack) Flynn JM, Skaggs DL, Waters PM. eds. Rockwood and Wilkin's Fractures in children. $8^{\text {th }}$ edn. Philadelphia, PA: Wolters Kluwer Health 2015:652-700.

[14] Inoue G, Horii E. Case report. Combined shear fractures of the trochlea and capitellum associated with the anterior fracture-dislocation of the elbow. J Orthop Trauma 1992;6(3):373-5.

[15] Borris LC, Lassen MR, Christensen CS. Elbow dislocation in children and adults. A long-term followup of conservatively treated patients. Acta Orthop Scand 1987;58(6):649-51.

[16] Skak SV, Olsen SD, Smaabrekke A. Deformity after fracture of the lateral humeral condyle in children. J Ped Orthop B 2001;10(2):142-52.

[17] Stans AA, Maritz NG, O’Driscoll SW, et al. Operative treatment of elbow contracture in patients twenty-one years of age or younger. J Bone Joint Surg Am 2002;84A(3):382-7.

[18] Farsetti P, Potenza V, Caterini R, et al. Long-term results of treatment of fractures of the medial humeral epicondyle in children. J Bone Joint Surg Am 2001;83A(9):1299-305.

[19] Gausepohl T, Mader K, Pennig D. Mechanical distraction for the treatment of post-traumatic stiffness of the elbow in children. J Bone Joint Surg Am 2006;88(5):1011-21.

[20] Mader K, Koslowsky TC, Gausepohl T, et al. Mechanical distraction for the treatment of posttraumatic stiffness of the elbow in children and adolescents: surgical technique. J Bone Joint Surg Am 2007;89(Suppl 2 Pt 1):26-35. 
[21] Buckwalter JA. Should bone, soft tissue and joint injuries be treated with rest of activity? J Orthop Res 1995;13(2):155-6.

[22] Eygendaal D, Olsen BS, Jensen SL, et al. Kinematics of partial and total ruptures of the medial collateral ligament of the elbow. J Shoulder Elbow Surg 1999;8(6):612-6.

[23] Cheng JC, Shen WY. Limb fracture pattern in different pediatric age groups: a study of 3,350 children. J Orthop Trauma 1993;7(1):15-22.
[24] Zionts LE, Mirzayan R. Elbow stiffness following malunion of a fracture of the lateral epicondyle of the humerus in a child: a case report. J Bone Joint Surg Am 2002;84-A(5):818-21.

[25] El Assil 0, Tatar M, Uzel AP. Floating forearm with pure dislocations. Hand Surgery and Rehabilitation 2016;35(3):225-8. 